\title{
Optically opaque color-flavor locked phase inside compact stars
}

\author{
Igor A. Shovkovy ${ }^{1,2, *}$ and Paul J. Ellis ${ }^{2}$ \\ ${ }^{1}$ Institut für Theoretische Physik, Johann Wolfgang Goethe-Universität, 60054 Frankfurt/Main, Germany \\ ${ }^{2}$ School of Physics and Astronomy, University of Minnesota, Minneapolis, Minnesota 55455
}

(October 25, 2018)

\begin{abstract}
The contribution of thermally excited electron-positron pairs to the bulk properties of the color-flavor locked quark phase inside compact stars is examined. The presence of these pairs causes the photon mean free path to be much smaller than a typical core radius $\left(R_{0} \simeq 1 \mathrm{~km}\right)$ for all temperatures above $25 \mathrm{keV}$ so that the photon contribution to the thermal conductivity is much smaller than that of the Nambu-Goldstone bosons. We also find that the electrons and positrons dominate the electrical conductivity, while their contributions to the total thermal energy is negligible.
\end{abstract}

PACS number(s): 26.60.+c, 12.38.Aw, 21.65.+f

While at present there is no unequivocal evidence that free quarks exist in compact stars, it is important to examine the role they might play since the observational situation may well change in the future. If quarks are present in the center of compact stars, it is most likely that they will be found in the color-flavor locked (CFL) phase [1]. There exists a rather detailed understanding of the basic properties of CFL quark matter [1-7]. This brief report augments our previous discussion [8] of the thermal properties of CFL quark matter by addressing the role of thermally excited electron-positron pairs which, hitherto, has been overlooked.

We start by mentioning that it is now commonly accepted that the CFL phase is electrically neutral [9]. This would suggest that the chemical potential related to the electric charge, $\mu_{e}$, is zero; implying that at finite temperature the (nonvanishing) densities of electrons and positrons are equal. In fact, Lorentz invariance is broken in the CFL phase and the positively and negatively charged kaons differ in mass, as do pions [10]. Therefore, at finite temperature, the densities of the positively and negatively charged species are not exactly the same; and this must be balanced by differing electron and positron densities in order to maintain charge neutrality. Thus $\mu_{e}$ is nonzero. However, at temperatures below $5 \mathrm{MeV}, \mu_{e}$ drops rapidly to zero [11], and it is completely negligible at temperatures of $1 \mathrm{MeV}$ or less, which are our principal interest here. It is therefore sufficient to set $\mu_{e}=0$ in assessing the impact of electron-positron pairs on the

\footnotetext{
${ }^{*}$ On leave from Bogolyubov Institute for Theoretical Physics, 03143 Kiev, Ukraine.
}

physical properties of CFL quark matter.

The first issue to address is the photon mean free path. Since photons scatter quite efficiently from charged leptons, even small numbers of electrons and positrons could substantially reduce the transparency of CFL quark matter in the core of a compact star. Now the photon mean free path can be rather well approximated by the simple expression

$$
\ell_{\gamma} \simeq \frac{1}{2 n_{e}(T) \sigma_{\mathrm{T}}},
$$

where $n_{e}(T)$ is the equilibrium density of electrons in a plasma at temperature $T$, the factor 2 takes into account the equal density of positrons, and

$$
\sigma_{\mathrm{T}}=\frac{8 \pi}{3} \frac{\alpha^{2}}{m_{e}^{2}} \approx 66.54 \mathrm{fm}^{2}
$$

is the well-known expression for the Thomson cross section in terms of the fine structure constant $\alpha$ and the electron mass $m_{e}$. This expression is the limiting case of the more complicated Compton cross section for low photon energies. Since this limit works rather well for $\omega_{\gamma} \ll m_{e}$ and $m_{e} \simeq 0.5 \mathrm{MeV}$, this is sufficient for the purposes of this paper.

The average equilibrium electron or positron density in a finite temperature neutral plasma [12] is

$$
n_{e}=\frac{m_{e}^{2} T}{\pi^{2}} \sum_{k=1}^{\infty} \frac{(-1)^{k+1}}{k} K_{2}\left(\frac{m_{e} k}{T}\right),
$$

where $K_{2}$ is a modified Bessel function. By making use of this result we find the temperature dependence of the photon mean free path in the CFL phase shown in Fig. 1. We see that $\ell_{\gamma} \lesssim 220 \mathrm{~m}$ for $T \gtrsim 25 \mathrm{keV}$. Since the radius of the CFL core $\left(R_{0}\right)$ is of order $1 \mathrm{~km}$, the photon mean free path is short for temperatures above $25 \mathrm{keV}$, so that the quark core of a compact star is opaque to light. Conversely, transparency can be considered to set in when the mean free path exceeds $1 \mathrm{~km}$, which occurs for temperatures below $23.4 \mathrm{keV}$.

Photons together with massless Nambu-Goldstone (NG) bosons $\phi$, which arise from the breaking of the global baryon number symmetry, dominate the thermal conductivity. The estimates above indicate that for $T \lesssim 25 \mathrm{keV}$, these components are equally important, as discussed in our previous study [8]. This is no longer true at higher temperatures since the photon contribution becomes negligible. Thus the thermal conductivity can be approximated, 


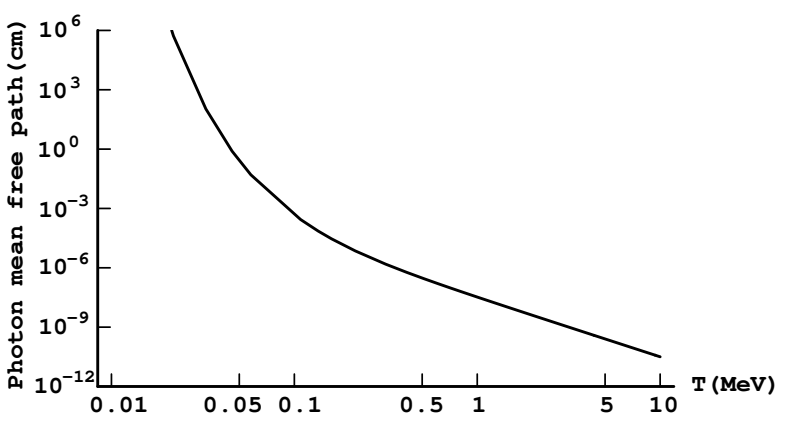

FIG. 1. Photon mean free path as a function of temperature in the color-flavor locked phase.

$$
\kappa_{\mathrm{CFL}} \simeq \kappa_{\phi} \simeq \frac{2 \pi^{2}}{15} T^{3} R_{0} \quad \text { for } \quad T \gtrsim 25 \mathrm{keV}
$$

where for the velocity we used $v_{\phi}=1 / \sqrt{3}$. Numerically, this leads to the following estimate

$$
\kappa_{\mathrm{CFL}} \simeq\left(7.2 \times 10^{31}\right) T_{\mathrm{MeV}}^{3} R_{0, \mathrm{~km}} \mathrm{erg} \mathrm{cm}{ }^{-1} \mathrm{sec}^{-1} \mathrm{~K}^{-1},
$$

where the notation indicates that $T$ is measured in units of $\mathrm{MeV}$ and $R_{0}$ is measured in units of $\mathrm{km}$. This differs from our previous result [8] by a factor $3 / 5$, which does not change the qualitative conclusion that the thermal conductivity of CFL matter is extremely high.

We also need to evaluate the electron-positron contribution to the thermal energy of the CFL core since this is relevant to the cooling. It is straightforward to obtain the expression (see Ref. [12])

$$
\begin{aligned}
E_{e}(T) & =\frac{8 T\left(R_{0} m_{e}\right)^{3}}{3 \pi} \sum_{k=1}^{\infty} \frac{(-1)^{k+1}}{k} \\
& \times\left[K_{1}\left(\frac{m_{e} k}{T}\right)+\frac{3 T}{m_{e} k} K_{2}\left(\frac{m_{e} k}{T}\right)\right] .
\end{aligned}
$$

In Fig. 2 we indicate this contribution to the thermal energy by a dashed line, while our previously calculated thermal energy due to the massless states, $E_{\phi, \gamma}$, is indicated by the solid line (the radius of the CFL quark core, $R_{0}$, is taken to be $1 \mathrm{~km}$ ). The comparison shows that the charged lepton contribution is very small, $E_{e} \ll E_{\phi, \gamma}$, for all temperatures below $0.1 \mathrm{MeV}$. Moreover, even at higher temperatures, $E_{e} \lesssim E_{\phi, \gamma}$. Thus, the presence of thermally excited electron-positron pairs does not qualitatively affect the thermal energy of CFL matter. This finding, together with the previous discussion of the thermal conductivity, means that the cooling mechanism described in Ref. [8] remains unchanged.

A separate issue is the electrical conductivity of CFL matter. Since electrons and positron are charged particles, they should give a nonvanishing contribution to this transport coefficient. In principle, charged pseudoNG bosons will also contribute, as mentioned in Ref. [8].
However, since even the lightest $K^{+}$boson has an estimated mass that is an order of magnitude larger than that of the electron (e.g., see Refs. [3,13]), these contributions will be strongly suppressed and electrons and positrons will dominate the electrical conductivity.

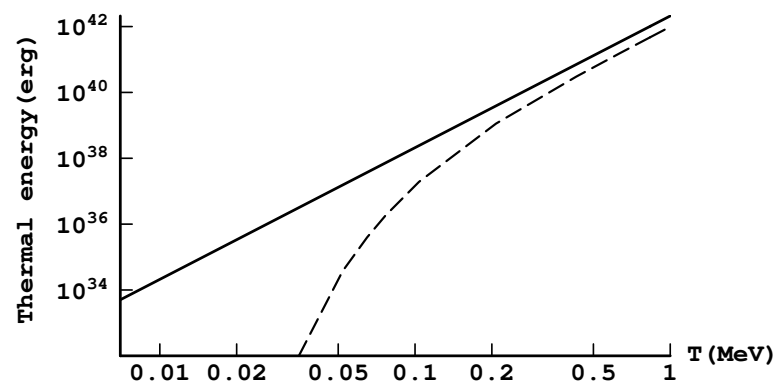

FIG. 2. The contributions of massless states (solid line) and charged leptons (dashed line) to the thermal energy of a CFL quark core with radius $R_{0}=1 \mathrm{~km}$.

In order to estimate the electrical conductivity of the thermally excited electron-positron plasma in the CFL phase, we need to know the temperature dependence of the electron mean free path. The mean free path is finite due to the scattering of electrons by positrons and other electrons, as well as annihilation, braking radiation (bremsstrahlung), and Compton scattering. These processes have recently been studied in the ultrarelativistic limit in order to determine the kinetic properties of gauge theories [14]. However, in the non-relativistic limit, the annihilation, braking radiation, and Compton scattering contributions are expected to be negligible, and the corresponding expression for the electron mean free path is known in the so-called leading logarithm approximation. This is sufficient if we restrict ourselves to temperatures $T \lesssim m_{e}$, in which case the electron mean free path [15] is

$$
\ell_{e} \sim \frac{T^{2}}{4 \pi \alpha^{2} n_{e} L_{e}}
$$

where $n_{e}$ is the number density of positrons which, according to our assumption, is the same as that of electrons given in Eq. (3). The so-called Coulomb logarithm $L_{e}$ appears as a result of the long range nature of the Coulomb interaction. Its value is determined by the Debye screening mass $m_{D}$ inside the plasma [15]:

$$
L_{e} \simeq \ln \frac{T}{m_{D} \max \left(\alpha, \bar{v}_{e}\right)}, \quad \text { where } \quad m_{D}^{2}=\frac{8 \pi \alpha n_{e}}{T},
$$

and $\bar{v}_{e} \simeq \sqrt{T / m_{e}}$ is the average thermal velocity of the electrons.

Now, to estimate the electrical conductivity of the electron-positron plasma existing inside the CFL quark core of the star, we use the following classical expression: 


$$
\sigma_{e} \simeq \frac{8 \pi \alpha n_{e} \ell_{e}}{m_{e} \bar{v}_{e}}
$$

relating the conductivity (in Heaviside-Lorentz units) to the electron mean free path $\ell_{e}$. If $\ell_{e}$ is less than the typical size of the CFL quark phase, $R_{0} \simeq 1 \mathrm{~km}$, which is the case for temperatures higher than about $17 \mathrm{keV}$, this expression for the electrical conductivity reads

$$
\sigma_{e} \sim \frac{2 T^{3 / 2}}{\alpha \sqrt{m_{e}} L_{e}} .
$$

It should be noted that this expression depends on the density of electrons only through the Coulomb logarithm $L_{e}$. If this expression and the ultrarelativistic result [14] are extrapolated to $T \sim m_{e}$, it is satisfactory that they are of similar magnitude.

At lower temperatures $(T \lesssim 17 \mathrm{keV})$, on the other hand, the electrical conductivity in Eq. (9) becomes

$$
\sigma_{e} \simeq \frac{2^{5 / 2} \alpha m_{e} T R_{0}}{\sqrt{\pi}} \exp \left(-\frac{m_{e}}{T}\right)
$$

This makes it clear that the contribution from, say, a positively charged kaon will be enormously suppressed due to a factor $\exp \left(-m_{K^{+}} / T\right)$.

Note that the contribution of the electrons and positrons themselves to the thermal conductivity is always small compared to the contribution of NG bosons in Eq. (4). At temperatures higher than about $17 \mathrm{keV}$, it is small because the electron mean free path is small, while at lower temperatures $\ell_{e}$ is restricted by the size of the core, and the specific heat decreases exponentially with decreasing temperature.

In conclusion, we have shown that the presence of a thermally excited electron-positron plasma inside the neutral CFL core of a compact star has some interesting consequences. One of the most interesting, and somewhat unexpected, consequences is that CFL quark matter is optically opaque at temperatures higher than about $25 \mathrm{keV}$. This suppresses the photon contribution to the thermal conductivity, although at lower temperatures it is comparable to that arising from the massless NG boson, $\phi$. Nevertheless, the thermal conductivity of CFL matter is very large in all regimes, so that the thermal energy from the CFL core of a compact star is efficiently conducted away to the outer nuclear layer and the core remains nearly isothermal [8]. If bare CFL quark stars exist in the Universe, the fact that they only become transparent to photons when they have cooled to rather low temperatures might be of some observational importance. A similar interplay between NG boson and photon contributions should also appear in other transport coefficients, such as the shear viscosity.

The electrical conductivity of the CFL phase will be largely determined by the electron-positron plasma since contributions from other charged particles, such as kaons, are strongly suppressed. This may turn out to be important in studying the dynamo mechanism [16] of magnetic field generation in compact stars with quark cores.

We thank Thomas Schäfer for comments on the first version of the paper. This work was supported by the U.S. Department of Energy Grant No. DE-FG0287ER40328. The work of I.A.S. was partially supported by the Gesellschaft für Schwerionenforschung (GSI) and by the Bundesministerium für Bildung und Forschung (BMBF).

[1] M. Alford, K. Rajagopal, and F. Wilczek, Nucl. Phys. B537, 443 (1999).

[2] R. Casalbuoni and R. Gatto, Phys. Lett. B 464, 111 (1999).

[3] D.T. Son and M.A. Stephanov, Phys. Rev. D 61, 074012 (2000); 62, 059902(E) (2000).

[4] I.A. Shovkovy and L.C.R. Wijewardhana, Phys. Lett. B 470, 189 (1999); T. Schafer, Nucl. Phys. B575, 269 (2000).

[5] D.H. Rischke, Phys. Rev. D 62, 054017 (2000).

[6] C. Manuel and M.H.G. Tytgat, Phys. Lett. B 501, 200 (2001); V.A. Miransky, I.A. Shovkovy, and L.C.R. Wijewardhana, Phys. Rev. D 63, 056005 (2001).

[7] K. Rajagopal and F. Wilczek, in At the Frontier of Particle Physics: Handbook of QCD, edited by M. Shifman (World Scientific, Singapore, 2001), Vol. 3, p.2061; D.K. Hong, Acta Phys. Pol. B 32, 1253 (2001); M.G. Alford, Annu. Rev. Nucl. Part. Sci. 51, 131 (2001).

[8] I.A. Shovkovy and P.J. Ellis, Phys. Rev. C 66, 015802 (2002); astro-ph/0207346.

[9] K. Rajagopal and F. Wilczek, Phys. Rev. Lett. 86, 3492 (2001); A.W. Steiner, S. Reddy, and M. Prakash, Phys. Rev. D 66, 094007 (2002); F. Neumann, M. Buballa, and M. Oertel, Nucl. Phys. A714, 481 (2003).

[10] P.F. Bedaque and T. Schäfer, Nucl. Phys. A697, 802 (2002).

[11] S. Reddy, M. Sadzikowski, and M. Tachibana, Nucl. Phys. A714, 337 (2003).

[12] S.M. Johns, P.J. Ellis, and J.M. Lattimer, Astrophys. J. 473, 1020 (1996).

[13] T. Schafer, Phys. Rev. D 65, 094033 (2002).

[14] G. Baym, H. Monien, C.J. Pethick, and D.G. Ravenhall, Phys. Rev. Lett. 64, 1867 (1990); G. Baym and H. Heiselberg, Phys. Rev. D 56, 5254 (1997); P. Arnold, G.D. Moore, and L.G. Yaffe, J. High Energy Phys. 0011, 001 (2000).

[15] E.M. Lifshitz and L.P. Pitaevskii, Physical kinetics (Pergamon, New York, 1981).

[16] C. Thompson and R.C. Duncan, Astrophys. J. 408, 194 (1993). 